\title{
The Riemann Zeta Function of a Matrix/Tensor
}

\author{
Bohua $\operatorname{Sun}^{1}$ \\ ${ }^{1}$ Cape Peninsula University of Technology, Cape Town, South Africa*
}

(Dated: September 30, 2018)

This paper attempts to extend the Riemann Zeta function of a complex number to a function of a matrix and/or a tensor $\boldsymbol{A}$, namely

$$
\zeta(\boldsymbol{A})=\sum_{n=1}^{\infty} \frac{1}{n^{\boldsymbol{A}}}=\sum_{n=1}^{\infty} \sum_{k=1}^{n} \lambda_{k} \boldsymbol{A}^{k}
$$

and inverse

$$
\boldsymbol{A}=\sum_{n=1}^{\infty} \sum_{k=1}^{n} \mu(n) \lambda_{k} \zeta\left(\boldsymbol{A}^{k}\right)
$$

where $\mu(n)$ is the Möbius function, $\boldsymbol{A}$ is a complex matrix or tensor with any order, and $\lambda_{k}$ is eigenvalue of the matri/tensor $\boldsymbol{A}$. This kind of calculations on the Riemann Zeta function has never been seen in the literature. Some examples are provided.

Keywords: Riemann Zeta function, matrix, tensor

\section{THE RIEMANN ZETA FUNCTION AND THE RIEMANN HYPOTHESIS}

Natural numbers form the basis of our arithmetic, with various operations defined among these numbers. All of us learn to use four basic operations: addition, subtraction, multiplication and division. The latter, division, hides one of the most enigmatic internal structures of the set of the natural numbers, namely that there are special numbers, the primes, among the natural numbers which cannot be divided by any other natural number, other than unity and themselves, without a remainder. Euclid of Alexandria proved that there are infinitely many such numbers. Later, Eratosthenes of Cyrene gave a theoretical algorithm, a sieve, for finding these primes amongst the natural numbers. Despite all efforts in the last two thousand years, the efficient determination as to whether a given number is prime or not still proves a remarkable challenge [1-3].

The Riemann zeta function $\zeta(s)$ is a function of a complex variable $s=\sigma+i t$. (The notation $s, \sigma$ and $t$ is used traditionally in the study of the zeta function, following Riemann.) The following infinite series converges for all complex numbers $s$ with real part greater than 1 , and defines $\zeta(s)$ in this case:

$$
\zeta(s)=\sum_{n=1}^{\infty} \frac{1}{n^{s}}=\frac{1}{1^{s}}+\frac{1}{2^{s}}+\frac{1}{3^{s}}+\cdots \quad \sigma=\operatorname{Re}(s)>1 .
$$

Via analytic continuation, one can show that: $\zeta(-1)=$ $-\frac{1}{12}, \zeta(0)=-\frac{1}{2}, \zeta(1)=\infty$, and $\zeta(2)=\frac{\pi^{2}}{6}$.

Bernhard Riemann, who was the first to apply the tools of complex analysis to this function in Eq.(1), proved that the function defined by the infinite summation (Riemann, 1859) can be analytically continued over the complex $s$ plane, except for $s=1$. This analytic continuation of the function is called the Riemann-zeta function. Here we follow the traditional notation, with s denoting a complex number, $s=\sigma+i t$, where $\sigma$ and $t$ are real numbers and $i$ is the usual imaginary unit. Riemann also derived a functional equation, containing the $\zeta(s)$ function, which is valid for all complex $\mathrm{s}$ and exhibits mirror symmetry around the $\sigma=1 / 2$ vertical line, called the critical line. Figure 1 depicts the pole and zero structure of $\zeta(s)$ on the complex $s$ plane including the possible zeros off the critical line.

So far the statements about the zeros of $\zeta$ (s) and their locations on the complex plain were simple. However the distribution of the non-trivial zeros holds one of the most intriguing and enigmatic mathematical mysteries of the last century and a half. It is embarrassingly easy to pose Riemann' s conjecture:

The Riemann Conjecture All non-trivial zeros of $\zeta(s)$ have the form $\sigma=1 / 2+i t$, where $t$ is a real number.

In other words all non-trivial zeros lie on the critical line. In 1900 Hilbert nominated the Riemann Hypothesis as the eighth problem on his famous list of compelling problems in mathematics (Hilbert, 1902). Since then not just professional mathematicians but mathematical soldiers of fortune tried, and still try, to verify its validity. 


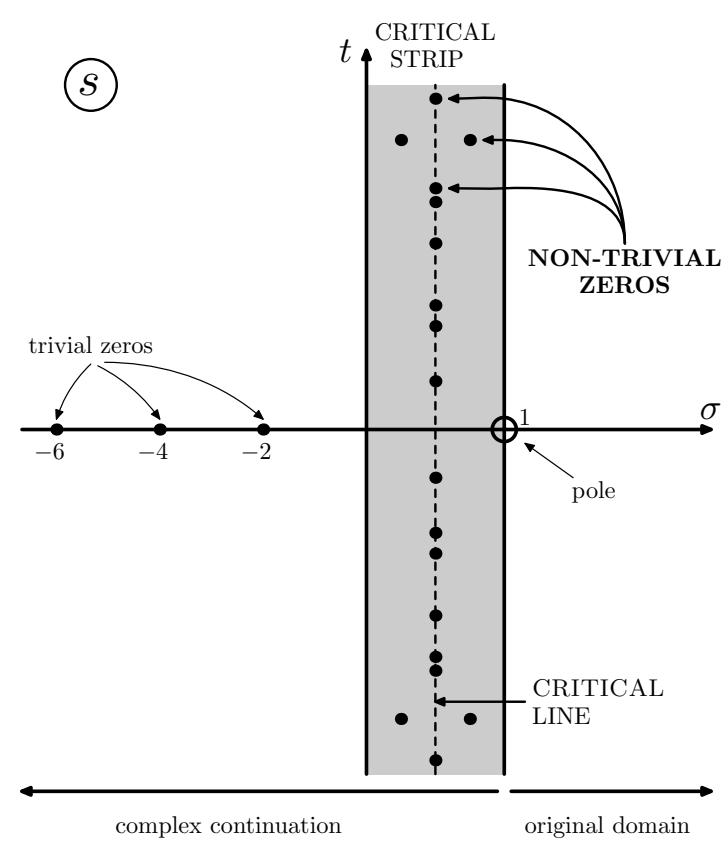

FIG. 1: The Riemann Zeta On Complex Plane

The stakes are high. Whoever proves or disproves this hypothesis engraves his name in the tablets of the history of mathematics, and may also receive one million dollars from the Clay Mathematics Institute [4].

\section{CAYLEY - HAMILTON THEOREM}

In linear algebra, the Cayley - Hamilton theorem (named after the mathematicians Arthur Cayley and William Rowan Hamilton) states that every square matrix over a commutative ring (such as the real or complex field) satisfies its own characteristic equation.

Consider a square matrix $\boldsymbol{A}$ with dimension n and with a characteristic polynomial

$$
\triangle(\lambda)=|\boldsymbol{A}-\lambda \boldsymbol{I}|=\lambda^{n}+c_{n-1} \lambda^{n-1}+\cdots+c_{0} .
$$

and define a corresponding matrix polynomial, formed by substituting $\boldsymbol{A}$ for $\lambda$ above

$$
\triangle(\boldsymbol{A})=\boldsymbol{A}^{n}+c_{n-1} \boldsymbol{A}^{n-1}+\cdots+c_{0} .
$$

where $\boldsymbol{I}$ is the identity matrix. The Cayley-Hamilton theorem states that every matrix satisfies its own characteristic equation, that is

$$
\triangle(\boldsymbol{A})=\boldsymbol{A}^{n}+c_{n-1} \boldsymbol{A}^{n-1}+\cdots+c_{0}=[\mathbf{0}] .
$$

where $[\mathbf{0}]$ is the null matrix.

The Cayley-Hamilton theorem can be used to reduce the Order of a polynomial in a matrix/tensor, and to determine analytic functions of a matrix/tensor. Clearly, for a $\mathrm{n}$ order matrix $\boldsymbol{A}$, who has n eigenvalues, namely $\lambda_{n}, f(\boldsymbol{A})$, the function of the matrix $\boldsymbol{A}$, can be expressed as follows:

$$
f(\boldsymbol{A})=\sum_{k=1}^{n} \lambda_{k} \boldsymbol{A}^{k}
$$

Example: Find $e^{s \boldsymbol{A}}$, where $\boldsymbol{A}=\left[\begin{array}{cc}0 & 1 \\ -1 & 0\end{array}\right]$

For $\boldsymbol{A}, \triangle(\lambda)=|\boldsymbol{A}-\lambda \boldsymbol{I}|=\lambda^{2}+1=0$, giving $\lambda_{0}=i$ and $\lambda_{1}=-i$. Since $\boldsymbol{A}$ is the 2nd order matrix, then $e^{s A}=\alpha_{0} \boldsymbol{I}+\alpha_{1} \boldsymbol{A}$, thus

$$
\begin{aligned}
e^{i s} & =\alpha_{0}+i \alpha_{1}, \\
e^{-i s} & =\alpha_{0}-i \alpha_{1} .
\end{aligned}
$$

Notice $e^{i s}=\cos (s)+i \sin (s)$, we obtain $\alpha_{0}=\cos (s)$ and $\alpha_{1}=\sin (s)$. Then

$$
e^{s A}=\boldsymbol{I} \cos (s)+\boldsymbol{A} \sin (s)=\left[\begin{array}{cc}
\cos s & \sin s \\
-\sin s & \cos s
\end{array}\right] \text {. }
$$

\section{THE RIEMANN ZETA FUNCTION OF A MATRIX OR A TENSOR}

The Riemann Zeta function of a matrix/tensor can be defined as follows $\zeta(s)$ in this case:

$$
\zeta(\boldsymbol{A})=\sum_{n=1}^{\infty} \frac{1}{n^{\boldsymbol{A}}}=\frac{1}{1^{\boldsymbol{A}}}+\frac{1}{2^{\boldsymbol{A}}}+\frac{1}{3^{\boldsymbol{A}}}+\cdots .
$$

From the Cayley-Hamilton theorem, every term of the above function can be expressed as follows

$$
\frac{1}{n^{\boldsymbol{A}}}=\sum_{k=1}^{n} \lambda_{k} \boldsymbol{A}^{k}
$$

where $\boldsymbol{A}$ is a $n \times n$ matrix and $\lambda_{k}$ is eigenvalue of the matrix. Therefore, we have the Riemann Zeta function of a matrix

$$
\zeta(\boldsymbol{A})=\sum_{n=1}^{\infty} \frac{1}{n^{\boldsymbol{A}}}=\sum_{n=1}^{\infty} \sum_{k=1}^{n} \lambda_{k} \boldsymbol{A}^{k}
$$

If we know $\zeta(:)$ and looking for the matrix $\boldsymbol{A}$, the above relations can simplify be inverse as to follows

$$
\boldsymbol{A}=\sum_{n=1}^{\infty} \sum_{k=1}^{n} \mu(n) \lambda_{k} \zeta\left(\boldsymbol{A}^{k}\right)
$$

where the Möbius function $\mu(n)$ is defined as follows: $\mu(1)=1 ; \mu(n)=0$ if $n$ has a square divisor; and $\mu\left(p_{1} p_{2} \cdots p_{k}\right)=(-1)^{k}$ if all pi,s are different. Thus $\mu(2)=-1$ and $\mu(12)=\mu\left(2^{2} \times 3\right)=0, \mu(21)=\mu(3 \times 7)=$ $(-1)^{2}=1$, for more info please see Ref.[3, 5]. 


\section{APPLICATIONS}

In this section, we will demonstrate some applications of solution (10).

Pronlem 1 Find $n^{s \boldsymbol{A}}$, where $\boldsymbol{A}=\left[\begin{array}{cc}0 & 1 \\ -1 & 0\end{array}\right]$

For $\boldsymbol{A}, \triangle(\lambda)=|\boldsymbol{A}-\lambda \boldsymbol{I}|=\lambda^{2}+1=0$, giving $\lambda_{0}=i$ and $\lambda_{1}=-i$. Since $\boldsymbol{A}$ is the 2 nd order matrix, then $n^{s A}=\alpha_{0} \boldsymbol{I}+\alpha_{1} \boldsymbol{A}$, thus

$$
\begin{aligned}
n^{i s} & =\alpha_{0}+i \alpha_{1}, \\
n^{-i s} & =\alpha_{0}-i \alpha_{1} .
\end{aligned}
$$

Notice $n^{i s}=\cos (s \ln n)+i \sin (s \ln n)$, we obtain $\alpha_{0}=$ $\cos (s \ln n)$ and $\alpha_{1}=\sin (s \ln n)$. Then

$$
\begin{aligned}
n^{s \boldsymbol{A}} & =\boldsymbol{I} \cos (s \ln n)-\boldsymbol{A} \sin (s \ln n) \\
& =\left[\begin{array}{cc}
\cos (s \ln n) & \sin (s \ln n) \\
-\sin (s \ln n) & \cos (s \ln n)
\end{array}\right] .
\end{aligned}
$$

Pronlem 2 Find $n^{s \boldsymbol{A}}$, where $\boldsymbol{A}=\frac{1}{\ln n}\left[\begin{array}{cc}0 & 1 \\ -1 & 0\end{array}\right]$

For $\boldsymbol{A}, \triangle(\lambda)=|\boldsymbol{A}-\lambda \boldsymbol{I}|=\lambda^{2}+\left(\frac{1}{\ln n}\right)^{2}=0$, giving $\lambda_{0}=i /(\ln n)$ and $\lambda_{1}=-i /(\ln n)$. Since $\boldsymbol{A}$ is the 2 nd order matrix, then $n^{s A}=\alpha_{0} \boldsymbol{I}+\alpha_{1} \boldsymbol{A}$, thus

$$
\begin{aligned}
n^{i s} & =\alpha_{0}+i \alpha_{1}, \\
n^{-i s} & =\alpha_{0}-i \alpha_{1} .
\end{aligned}
$$

Notice $n^{i s /(\ln n)}=\cos (s)+i \sin (s)$, we obtain $\alpha_{0}=\cos (s)$ and $\alpha_{1}=(\ln n) \sin (s)$. Then

$$
\begin{aligned}
n^{s A} & =\boldsymbol{I} \cos (s)-\boldsymbol{A}(\ln n) \sin (s) \\
& =\left[\begin{array}{cc}
\cos (s) & \sin (s) \\
-\sin (s) & \cos (s)
\end{array}\right] .
\end{aligned}
$$

Pronlem 3 Find $n^{1 / 2+i \boldsymbol{A}}$, where $\boldsymbol{A}=\left[\begin{array}{cc}0 & 1 \\ -1 & 0\end{array}\right]$

For $\boldsymbol{A}, \triangle(\lambda)=|\boldsymbol{A}-\lambda \boldsymbol{I}|=\lambda^{2}+1=0$, giving $\lambda_{0}=i$ and $\lambda_{1}=-i$. Since $\boldsymbol{A}$ is the 2nd order matrix, then $n^{i \boldsymbol{A}}=\alpha_{0} \boldsymbol{I}+\alpha_{1} \boldsymbol{A}$, thus

$$
\begin{array}{r}
n^{-1}=\alpha_{0}+i \alpha_{1}, \\
n=\alpha_{0}-i \alpha_{1} .
\end{array}
$$

we obtain $\alpha_{0}=\left(n+n^{-1}\right) / 2$ and $\alpha_{1}=i\left(n-n^{-1}\right) / 2$. Then

$$
\begin{aligned}
n^{1 / 2+i \boldsymbol{A}} & =n^{1 / 2}\left[\frac{1}{2}\left(n+n^{-1}\right) \boldsymbol{I}-\frac{1}{2} i\left(n-n^{-1}\right) \boldsymbol{A}\right] \\
& =\frac{1}{2} n^{1 / 2}\left[n(\boldsymbol{I}-i \boldsymbol{A})+n^{-1}(\boldsymbol{I}+i \boldsymbol{A})\right] \\
& =\frac{1}{2} n^{1 / 2}\left[\begin{array}{cc}
n+n^{-1} & -i\left(n-n^{-1}\right) \\
i\left(n-n^{-1}\right) & n+n^{-1}
\end{array}\right] \\
& =\frac{1}{2} n^{-1 / 2}\left[\begin{array}{cc}
n^{2}+1 & -i\left(n^{2}-1\right) \\
i\left(n^{2}-1\right) & n^{2}+1
\end{array}\right] .
\end{aligned}
$$

Pronlem 4 Find $n^{-1 / 2 \boldsymbol{I}+i \boldsymbol{A}}$, where $\boldsymbol{A}=\left[\begin{array}{cc}0 & 1 \\ -1 & 0\end{array}\right]$

Set $\boldsymbol{B}=-1 / 2 \boldsymbol{I}+i \boldsymbol{A}=\left[\begin{array}{cc}-1 / 2 & i \\ -i & -1 / 2\end{array}\right]$.

For $\boldsymbol{B}, \triangle(\lambda)=|\boldsymbol{B}-\lambda \boldsymbol{I}|=(-1 / 2-\lambda)^{2}-1=0$, giving $\lambda_{0}=1 / 2$ and $\lambda_{1}=-3 / 2$. Since $\boldsymbol{B}$ is the 2 nd order matrix, then $n^{\boldsymbol{B}}=\alpha_{0} \boldsymbol{I}+\alpha_{1} \boldsymbol{B}$, thus

$$
\begin{aligned}
n^{-1} & =\alpha_{0}+i \alpha_{1}, \\
n & =\alpha_{0}-i \alpha_{1} .
\end{aligned}
$$

we obtain $\alpha_{0}=\frac{1}{4}\left(3 n^{1 / 2}+n^{-3 / 2}\right)$ and $\alpha_{1}=\frac{1}{2}\left(n^{1 / 2}-\right.$ $\left.n^{-3 / 2}\right)$. Then

$$
\begin{aligned}
& n^{-1 / 2+i \boldsymbol{A}} \\
& =\frac{1}{4}\left(3 n^{1 / 2}+n^{-3 / 2}\right) \boldsymbol{I}+\frac{1}{2}\left(n^{1 / 2}-n^{-3 / 2}\right) \boldsymbol{B} \\
& =\frac{1}{4}\left(3 n^{1 / 2}+n^{-3 / 2}\right)\left[\begin{array}{cc}
1 & 0 \\
0 & 1
\end{array}\right] \\
& -\frac{1}{4}\left(n^{1 / 2}-n^{-3 / 2}\right)\left[\begin{array}{cc}
1 & -2 i \\
2 i & 1
\end{array}\right] \\
& =\frac{1}{2}\left[\begin{array}{cc}
n^{1 / 2}+n^{-3 / 2} & i\left(n^{1 / 2}-n^{-3 / 2}\right) \\
-i\left(n^{1 / 2}-n^{-3 / 2}\right) & n^{1 / 2}+n^{-3 / 2}
\end{array}\right] .
\end{aligned}
$$

Pronlem 5 Find $n^{-1 / 2 I+i \sigma_{z}}$, where the Pauli matrix $\boldsymbol{\sigma}_{z}=\left[\begin{array}{cc}1 & 0 \\ 0 & -1\end{array}\right]$

Set $\boldsymbol{B}=-1 / 2 \boldsymbol{I}+i \boldsymbol{\sigma}_{z}=\left[\begin{array}{cc}i-1 / 2 & 0 \\ 0 & -i-1 / 2\end{array}\right]$.

For $\boldsymbol{B}, \triangle(\lambda)=|\boldsymbol{B}-\lambda \boldsymbol{I}|=(i-1 / 2-\lambda)(-i-1 / 2-\lambda)=$ 0 , giving $\lambda_{0}=i-1 / 2$ and $\lambda_{1}=-i-1 / 2$. Since $\boldsymbol{B}$ is the 2nd order matrix, then $n^{\boldsymbol{B}}=\alpha_{0} \boldsymbol{I}+\alpha_{1} \boldsymbol{B}$, thus

$$
\begin{gathered}
n^{i-1 / 2}=\alpha_{0}+(i-1 / 2) \alpha_{1}, \\
n^{-i-1 / 2}=\alpha_{0}+(-i-1 / 2) \alpha_{1} .
\end{gathered}
$$

we obtain $\alpha_{0}=n^{-i-1 / 2}-n^{i-1 / 2}$ and $\alpha_{1}=n^{-i-1 / 2}+(i+$ 
$1 / 2) n^{-i-1 / 2}-n^{i-1 / 2}$. Then

$$
\begin{aligned}
& n^{-1 / 2 \boldsymbol{I}+i \boldsymbol{\sigma}_{z}} \\
& =\frac{1}{2}\left[\begin{array}{cc}
(1++2 i)\left(n^{-i-1 / 2}-n^{i-1 / 2}\right) & 0 \\
0 & n^{-i-1 / 2}
\end{array}\right] .
\end{aligned}
$$

Pronlem 6 Calculate $\sum_{n=1}^{\infty} n^{-1 / 2 \boldsymbol{I}+i \boldsymbol{\sigma}_{z}}$, where the Pauli matrix $\boldsymbol{\sigma}_{z}=\left[\begin{array}{cc}1 & 0 \\ 0 & -1\end{array}\right]$

From problem 5, we have

$$
\begin{aligned}
& \sum_{n=1}^{\infty} n^{-1 / 2 \boldsymbol{I}+i \boldsymbol{\sigma}_{z}} \\
& =\frac{1}{2} \sum_{n=1}^{\infty}\left[\begin{array}{cc}
(1+2 i)\left(\frac{1}{n^{1 / 2+i}}-\frac{1}{n^{1 / 2-i}}\right) & 0 \\
0 & \frac{1}{n^{1 / 2+i}}
\end{array}\right] \\
& =\frac{1}{2}\left[\begin{array}{cc}
(1+2 i)[\zeta(1 / 2+i)-\zeta(1 / 2-i)] & 0 \\
0 & \zeta(1 / 2+i)
\end{array}\right]
\end{aligned}
$$

From Maple, we can get $\zeta(1 / 2+i)=0.1439364271-$ $0.7220997435 i$ and $\zeta(1 / 2-i)=0.1439364271+$ $0.7220997435 i$, thus we have

$$
\begin{aligned}
& \sum_{n=1}^{\infty} n^{-1 / 2 \boldsymbol{I}+i \boldsymbol{\sigma}_{z}} \\
& =\left[\begin{array}{cc}
1.444-0.722 i & 0 \\
0 & 0.0719-0.361 i
\end{array}\right] .
\end{aligned}
$$

Pronlem 7 Calculate $\sum_{n=1}^{\infty} n^{-1 / 2 I+i \sigma_{z}}$, where the unit tensor $\boldsymbol{I}=\boldsymbol{e}_{1} \otimes \boldsymbol{e}_{1}+\boldsymbol{e}_{2} \otimes \boldsymbol{e}_{2}$ and the Pauli tensor $\boldsymbol{\sigma}_{z}=\boldsymbol{e}_{1} \otimes \boldsymbol{e}_{1}-\boldsymbol{e}_{2} \otimes \boldsymbol{e}_{2}$, in which $\boldsymbol{e}_{1}, \boldsymbol{e}_{2}$ are the base vectors.

From problem 6, we have

$$
\begin{aligned}
& \sum_{n=1}^{\infty} n^{-1 / 2 \boldsymbol{I}+i \boldsymbol{\sigma}_{z}} \\
& =\frac{1}{2} \sum_{n=1}^{\infty}\left[(1+2 i)\left(\frac{1}{n^{1 / 2+i}}-\frac{1}{n^{1 / 2-i}}\right) \boldsymbol{e}_{1} \otimes \boldsymbol{e}_{1}\right. \\
& \left.+\frac{1}{n^{1 / 2+i}} \boldsymbol{e}_{2} \otimes \boldsymbol{e}_{2}\right]
\end{aligned}
$$

thus we have

$$
\begin{aligned}
& \sum_{n=1}^{\infty} n^{-1 / 2 \boldsymbol{I}+i \boldsymbol{\sigma}_{z}} \\
& =(1.444-0.722 i) \boldsymbol{e}_{1} \otimes \boldsymbol{e}_{1}+(0.0719-0.361 i) \boldsymbol{e}_{2} \otimes \boldsymbol{e}_{2}
\end{aligned}
$$

\section{CONCLUSIONS}

Any function of a matrix and tensor can be simplified by using the Cayley-Hamilton theorem, namely the spectrum representation of a this kind function can always be done if we can obtain the eigenvalue of the matrix/tensor. The study of the Riemann Zeta function of a matrix/tensor may help to understand the Riemann Hypothesis in the future.
[1] Riemann, B., 1859, Monatsber Berl Akad.

[2] Schumayer, D., and Hutchinson,D.A. W., Physics of the Riemann Hypothesis, Rev. Mod. Phys. 83:307-330,2011 DOI:10.1103/RevModPhys.83.307

[3] Hardy, G. H., and E. M. Wright, 1960, An Introduction to the Theory of Numbers (Clarendon Press) 4th edition. [4] https://en.wikipedia.org/wiki/Riemann_hypothesis

[5] Chen, N.X. Modified Möbius inverse formula and its applications in physics [J]. Phys.Rev.Lett. 64, 1193, 1990. 